\title{
Analysis of fetal and neonatal urine using proton nuclear magnetic resonance spectroscopy
}

\author{
P J D Foxall, S Bewley, G H Neild, C H Rodeck, J K Nicholson
}

\begin{abstract}
Aim-To use high field proton nuclear magnetic resonance spectroscopy $\left({ }^{1} \mathrm{H}\right.$ NMR) to characterise the low molecular weight metabolite composition of neonatal and fetal urine in relation to gestational age and perinatal outcome.

Methods-The first urine passed by two neonatal groups, six full term and five preterm infants with normal renal function, was analysed by ${ }^{1} \mathrm{H}$ NMR and compared with fetal urine from 14 cases with obstructive uropathy.

Results-The mean ratios of taurine, myo-inositol, and trimethylamine-Noxide (TMAO) to creatinine were $4 \cdot 3$, $10 \cdot 1$, and $14 \cdot 1$ times higher, respectively, in the preterm group when compared with those of the full term group. Fetal obstructive uropathy was characterised by glycosuria, amino and organic aciduria, regardless of gestational age (13-30 weeks). Conclusions-Samples of the first urine passed - that is, urine produced in fetal life - by normal preterm infants are useful controls for cases of obstructive uropathy detected in the third timester. ${ }^{1} H$ NMR will become a clinically useful tool for monitoring renal development and abnormalities in utero.

(Arch Dis Child 1995; 73: F153-F157)
\end{abstract}

Keywords: ${ }^{1} \mathrm{H}$ NMR spectroscopy, urinalysis, fetal obstructive uropathy, osmolytes.

The study of fetal renal development has major implications for understanding the aetiology of renal disorders in later life. ${ }^{1}$ The stages of development of normal renal function - that is, glomerular filtration and tubular reabsorption and secretion - remain poorly defined. Furthermore, there is little biochemical information relating to renal functional impairment associated with fetal obstructive uropathies. ${ }^{2-4}$ The lack of information on the time related changes of endogenous low molecular weight metabolites (such as amino acids, organic acids, amines and sugars) in human fetal urine is a reflection of both the limitations of the conventional analytical techniques used and the difficulty in obtaining normal urine in utero.

${ }^{1} \mathrm{H}$ NMR provides extensive information on both the structure and composition of low molecular weight metabolites in biological fluids and is a powerful, non-invasive, technique for exploring abnormal metabolic and toxicological processes. ${ }^{5}$ Human biofluids give characteristic ${ }^{1} \mathrm{H}$ NMR 'fingerprints' of metabolites that are affected by the basic mechanism, severity, and location of a pathological lesion..$^{5-13}$ An important advantage of ${ }^{1} \mathrm{H}$ NMR in the exploration of abnormal biochemistry is that metabolite pre-selection is not required, thereby eliminating a priori judgments by the investigator. Measurement time is short, less than 10 minutes for a standard one dimensional ${ }^{1} \mathrm{H}$ NMR experiment. Small sample volumes $(\sim 0.5 \mathrm{ml})$ can be used; this is an important pre-requisite for fetal and neonatal monitoring, and the unaltered sample can be further investigated by conventional methods. As such, ${ }^{1} \mathrm{H}$ NMR analysis of body fluids (including urine, blood plasma, and cerebrospinal fluid) has become of value in the clinical diagnosis and monitoring of inherited metabolic diseases, ${ }^{11-13}$ and the study of the biochemical environment of the fetus. ${ }^{14} 15$

In this preliminary study we used high field ${ }^{1} \mathrm{H}$ NMR spectroscopy to analyse neonatal and fetal urine, with the aim of generating new biochemical information on renal function in relation to gestational age and perinatal outcome.

\section{Methods}

Two groups of control neonatal urine samples were obtained: six full term healthy infants delivered by elective caesarean section (gestational age 38 weeks) and five healthy preterm infants (gestational age 25-36 weeks). To eliminate nutritional or xenobiotic influences on urinary biochemical composition, urine samples were collected into specimen bags shortly after birth and before oral or parenteral feeding or administration of any drugs. Fetal urine was collected by ultrasound guided transabdominal aspiration of the bladder from 14 cases of obstructive uropathy (gestational age 13-30 weeks). The birth outcome of the obstructive uropathy group was classified as follows: group 1, stillbirth or termination of pregnancy because of poor prognosis (seven fetuses, gestational age 13-22 weeks); group 2 , neonatal death with renal dysplasia on postmortem examination (two fetuses, gestational age 21 and 26 weeks); and group 3, alive with renal damage (five fetuses, gestational age 25-30 weeks). The study had local research ethics committee approval.

All urine samples were stored frozen at $-20^{\circ} \mathrm{C}$ after collection. Before NMR analysis, the urines were thawed at room temperature and centrifuged at $3000 \mathrm{rpm}$ for 10 minutes to remove any particulate matter. For each sample, $750 \mu \mathrm{l}$ was lyophilised and reconstituted in $750 \mu \mathrm{l}$ of deuterium oxide $\left(\mathrm{D}_{2} \mathrm{O}\right)$ containing $0.5 \mathrm{mM}$ sodium-3-trimethylsilyl$\left[2,2,3,3-{ }^{2} \mathrm{H}_{4}\right]-1$-propionate (TSP), which acted as chemical shift reference $(\delta 0.0)$ and internal 
standard for quantitation, and placed in a $5 \mathrm{~mm}$ NMR tube. Single pulse ${ }^{1} \mathrm{H}$ NMR measurements were made on a JEOL GSX500 spectrometer operating at $500 \cdot 14 \mathrm{MHz}^{1} \mathrm{H}$ frequency at ambient probe temperature.

Typically, 64 free induction decays were collected into 32768 computer points using $40^{\circ}$ pulses and a spectral width of $6000 \mathrm{~Hz}$. The data acquisition time per free induction decay was 2.73 seconds and a further delay of $2 \cdot 27$ seconds was added between the pulses to allow full $\mathrm{T}_{1}$ relaxation of metabolite protons. The residual water signal was suppressed by application of a gated secondary irradiation field (off during acquisition) at the water resonance frequency. Before Fourier transformation, an exponential apodisation function was applied to the free induction decay corresponding to a line broadening of $0.4 \mathrm{~Hz}$.

A

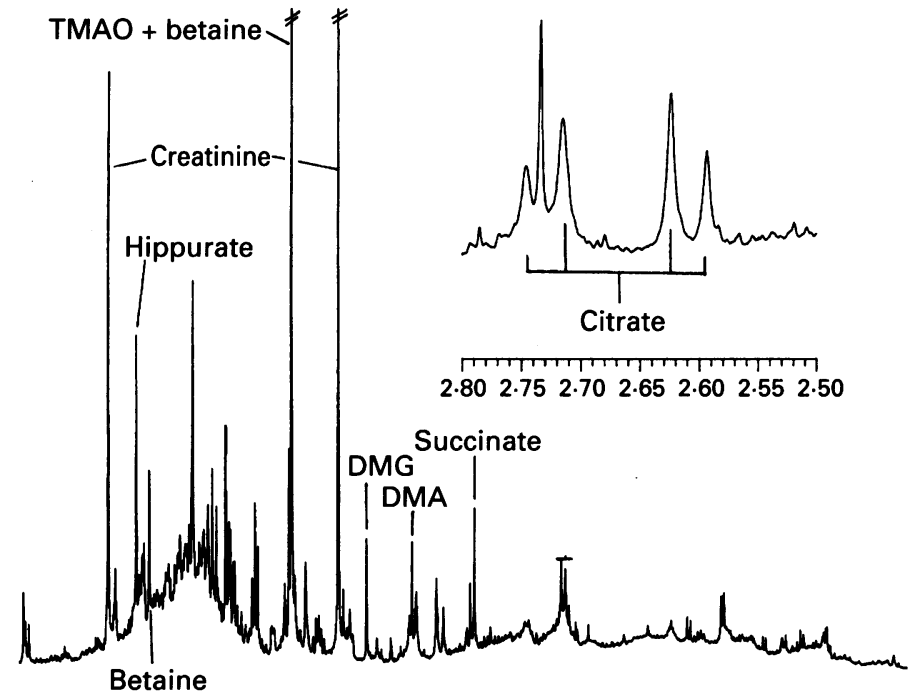

B

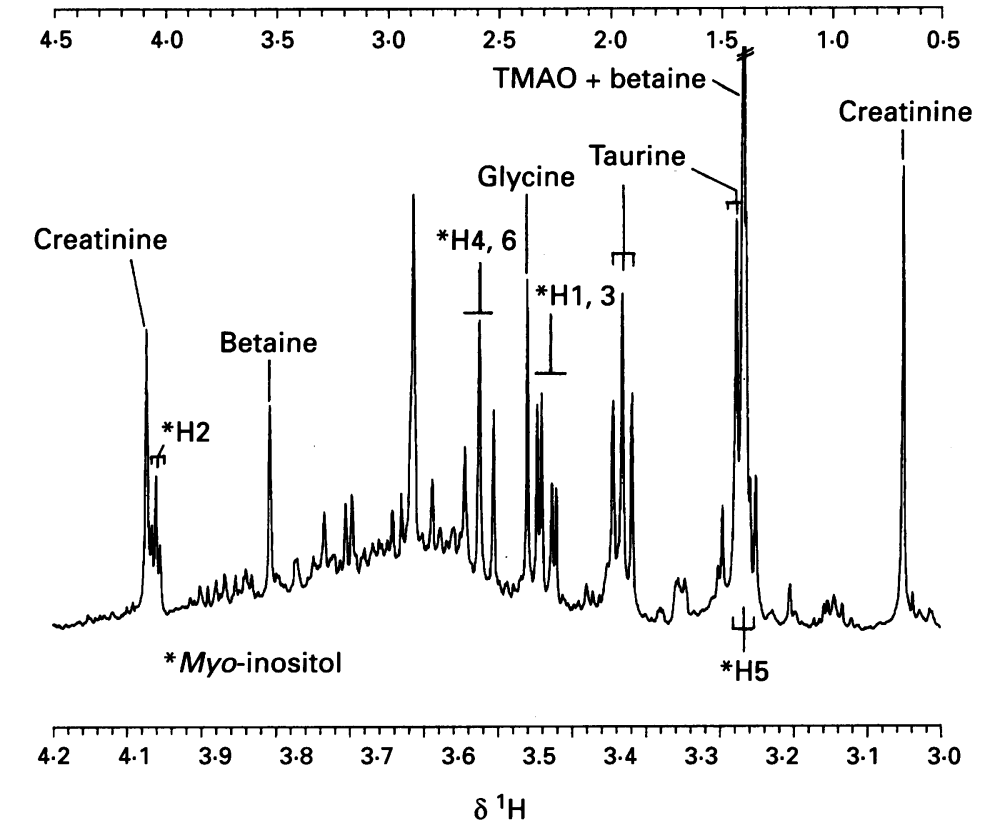

Figure 1 Partial $500 \mathrm{MHz}$ single pulse ${ }^{1} \mathrm{H}$ NMR spectrum of urine collected shortly after birth from: $(A)$ a normal full term infant, 38 weeks' gestation and, inset, the expanded $(\times 2$ vertical height) chemical shift region $\delta 2 \cdot 50-2 \cdot 80$ of the same spectrum to show the broadened resonances of citrate complexed with $\mathrm{Ca}^{2+}, \mathrm{Mg}^{2+}$ ions; (B) a normal pre-term infant, 33 weeks' gestation, chemical shift region $\delta 3 \cdot 0-4 \cdot 2 . D M A$, dimethylamine, $D M G$, dimethylglycine, NAC, N-acetyls of glycoproteins, TMAO, trimethylamine- $N$ oxide.
Assignment of resonances was made by consideration of chemical shift values relative to TSP, spin-spin coupling patterns, and the $\mathrm{pH}$ dependence of chemical shifts, as published before. ${ }^{6-10}$ All spectra were scaled to the same signal to noise ratio so that resonance intensities were directly comparable between urine samples. Quantitation of selected metabolite resonances was by peak height measurement relative to that of TSP and consideration of the number of protons contributing to the signals. ${ }^{6}$

\section{Results}

There were many similarities between the ${ }^{1} \mathrm{H}$ NMR profiles of the pre- and full term urines. The aliphatic region $(\delta 0.5-4.5)$ of the 500 $\mathrm{MHz}{ }^{1} \mathrm{H}$ NMR spectrum of urine collected from a full term infant (gestational age 38 weeks) is shown in fig $1 \mathrm{~A}$ and is representative of both neonatal groups. Sharp signals from creatinine, TMAO, betaine, taurine, succinate, dimethylglycine (DMG) and dimethylamine (DMA) were observed (fig 1A). We have previously assigned some of the singlet resonances in the $\delta 2 \cdot 0-2 \cdot 1$ region of the ${ }^{1} \mathrm{H}$ NMR spectrum to those of the $\mathrm{N}$-acetyl protons of mobile glycan side-chains of glycoprotein fragments (such as $\mathrm{N}$-acetylneuraminic acid). Broad citrate resonances were observed in the ${ }^{1} \mathrm{H}$ NMR spectra of all neonatal urines (fig 1A; inset). Addition of the metal chelating agent trisodium EDTA to a representative urine sample produced a sharpening of the citrate resonances and the appearance of signals for Ca-EDTA ${ }^{2-}$ and Mg-EDTA $^{2-}$ (data not shown). This indicated that the citrate broadening was mainly caused by complexation with $\mathrm{Ca}^{2+}$ and $\mathrm{Mg}^{2+}$ in the urine, involving chemical exchange of the metal ions with citrate at an intermediate rate on the NMR timescale. ${ }^{6}$

The notable differences between the ${ }^{1} \mathrm{H}$ NMR detected metabolite profiles of urine from pre- and full term infants are identified in fig $1 \mathrm{~B}$, which shows the spectral region $\delta 3.0$ to $\delta 4 \cdot 2$ of the ${ }^{1} \mathrm{H}$ NMR spectrum of urine from a pre-term infant (gestational age 33 weeks). The large resonance of the $\mathrm{N}-\mathrm{CH}_{3}$ group of TMAO at $\delta 3.27$ was overlapped with that of betaine at physiological $\mathrm{pH}$. Acidification of the sample resulted in a characteristic shift to high frequency of the signal supporting the assignment to that of TMAO. ${ }^{16}$ Betaine also gives a singlet for the $\mathrm{N}-\mathrm{CH}_{2}$ protons at $\delta 3.91$ which was chosen for metabolite quantitation because of minimal peak overlap with other species. Quantitative analysis of the ${ }^{1} \mathrm{H}$ NMR data showed that the excretion profiles of taurine, myo-inositol, and TMAO were higher in the pre-term group than the full term group (table 1). For comparison, the corresponding ratio for betaine is also given.

The ${ }^{1} \mathrm{H}$ NMR spectra of urine from the group with fetal obstructive uropathy were significantly different from those of the two control neonatal groups. The partial $500 \mathrm{MHz}$ ${ }^{1} \mathrm{H}$ NMR spectra of urine collected from one fetus in group 3 (30 weeks' gestation) and one fetus in group 1 (18 weeks' gestation) are shown in figs $2 A$ and $B$, respectively. In 
Table 1 Urinary betaine, taurine, myo-inositol and TMAO, expressed as the molar ratio to urine creatinine concentration for preterm and full term neonates, as measured by ${ }^{1} \mathrm{H}$ NMR spectroscopy

\begin{tabular}{lllll}
\hline & \multicolumn{4}{l}{ Metabolite: creatinine ratios } \\
\cline { 2 - 5 } Neonate group & Betaine & Taurine & myo-inositol & TMAOף \\
Pre-term $(\mathrm{n}=5)$ & $0.29(0.06)^{\star}$ & $1.04(0.05) \dagger$ & $1.31(0.34) \dagger$ & $0.42(0.08) \ddagger$ \\
Full-term $(\mathrm{n}=6)$ & $0.32(0.03)$ & $0.24(0.07)$ & $0.13(0.04)$ & $0.03(0.004)$ \\
\hline
\end{tabular}

Values represent mean ratios ( 1 standard error). Significance calculated using Student's unpaired $t$ test: ${ }^{\star}=$ no significant difference, $t=\mathrm{P}<0 \cdot 001, \neq=\mathrm{P}<0 \cdot 005$, when compared to full unpaired $t$ term group.

Corrected TMAO value, taking into consideration overlap of the signal with that of the betaine $\mathrm{N}-\mathrm{CH}_{2}$ singlet at $\delta 3 \cdot 27$, and calculated by subtraction of the betaine concentration calculated by measurement of the betaine $\mathrm{N}-\mathrm{CH}_{2}$ signal intensity at $\delta 3 \cdot 91$.

general, the ${ }^{1} \mathrm{H}$ NMR profiles of fetal urine were characterised by strong signals from glucose, amino acids, and organic acids. In contrast to all neonatal urines (fig 1), creatinine excretion was low, in relation to the other urinary metabolites, and the citrate resonances were sharper and seemed to be increased, indicating that the citrate was predominantly in the 'free' form - that is, in considerable excess over the divalent cation $\left(\mathrm{Ca}^{2+}, \mathrm{Mg}^{2+}\right)$ concentrations. High glucose concentrations were observed in all of the fetal urines analysed. Glycosuria was detected only in the urine of one preterm infant ( 25 weeks' gestation) and one full term infant ( 38 weeks' gestation). The $\delta 3.0$ to $\delta 4.0$ region of the standard one dimensional ${ }^{1} \mathrm{H}$ NMR spectra of fetal urine was particularly complex. Detailed assignment of this spectral region was difficult because of extensive overlap of signals from glucose (present at high concentrations) and those from other sugars, polyols (including myoinositol) and amino acids ( $\alpha-\mathrm{CH}$ resonances) which were present in lower concentrations. As a result, quantitation of taurine, myo-inositol, and TMAO for comparison with the neonatal groups would have been unreliable and was not performed. A summary of the low molecular weight metabolites, including chemical shift data, which give rise to the characteristic ${ }^{1} \mathrm{H}$ NMR profiles of the fetal and neonatal urines, are shown in table 2 .

\section{Discussion}

One aim of the study was to characterise the ${ }^{1} \mathrm{H}$ NMR urinary metabolite profiles in relation to gestational age. The ${ }^{1} \mathrm{H}$ NMR metabolite profiles of the two groups of preterm and full term neonatal urine were very similar in many respects (fig 1A). In humans nephrogenesis is complete by 34 weeks' gestation when the population of nephrons has reached its maximum. ${ }^{2}$ Postnatal renal maturation is essentially functional. The higher urinary excretion of creatinine and low excretion of glucose, amino, and organic acids observed in the ${ }^{1} \mathrm{H}$ NMR spectra of neonatal urine indicates maturation of glomerular filtration and tubular function. The urinary excretion of betaine was similar between the two neonatal groups studied, our ratios being comparable with those reported before. ${ }^{17}$ Urinary betaine increases in concentration during the neonatal period and is related to the metabolism of dietary choline. ${ }^{1718}$ The broadened signals from

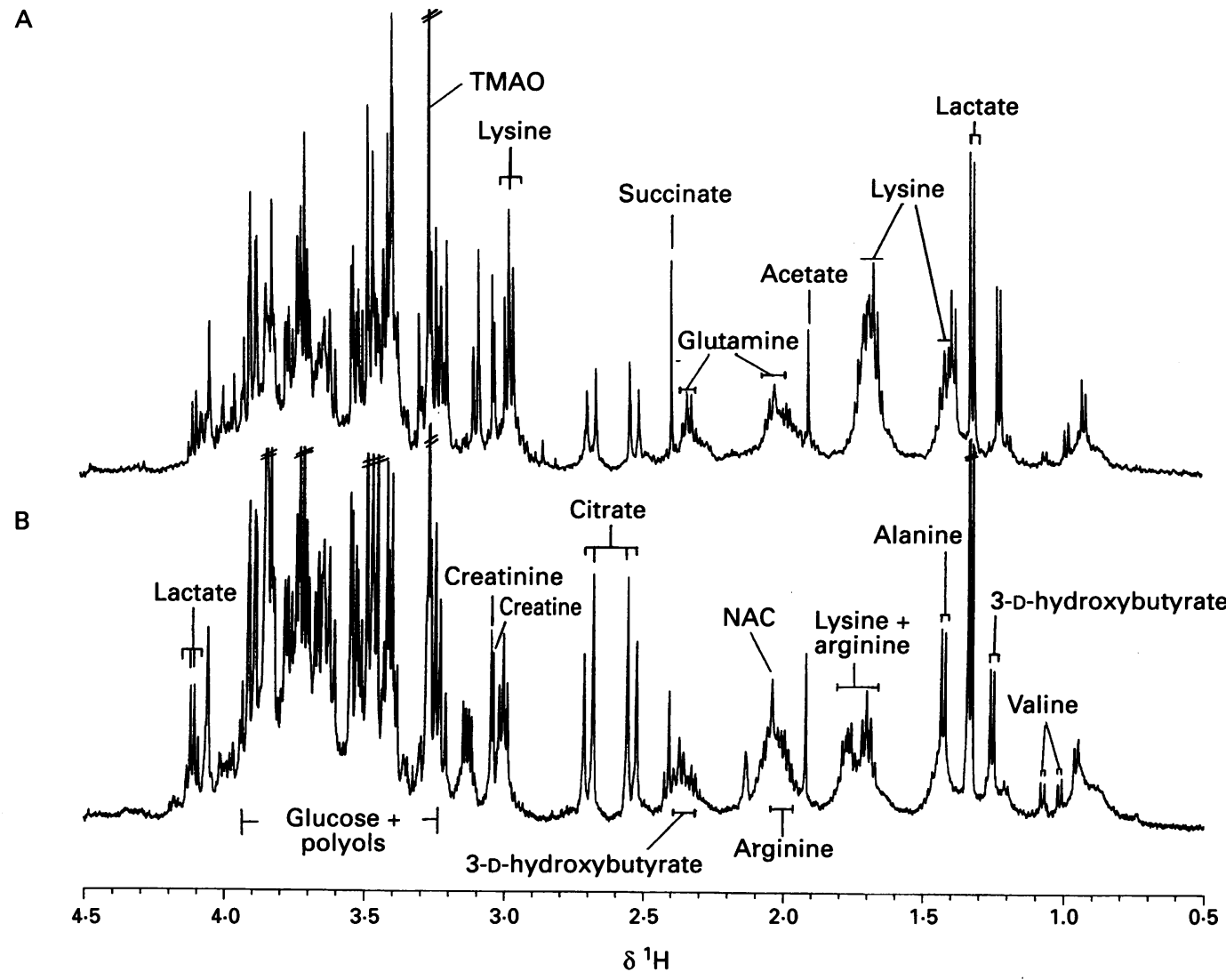

Figure 2 Partial $500 \mathrm{MHz}$ single pulse ${ }^{1} \mathrm{H}$ NMR spectra of urine obtained by needle aspiration of the fetal bladder from two cases of obstructive uropathy: (A) urine collected at 30 weeks' gestation from a fetus in group 3 and (B) urine collected at 18 weeks' gestation from a fetus in group 1. 
Table $2{ }^{1} H$ NMR spectral data of major metabolites detected in neonatal urine and fetal urine from cases of obstructive uropathy

\begin{tabular}{|c|c|c|}
\hline Group & Metabolite & $\delta p p m^{\star}$ and (multiplicity†) \\
\hline $\begin{array}{l}\text { FOU } \\
\text { FOU } \\
\text { FOU } \\
\text { FOU } \\
\text { FOU } \\
\text { FOU } \\
\text { FOU } \\
\text { FOU/PT/FT } \\
\text { FOU } \\
\text { FOU/PT/FT } \\
\text { FOU/PT/FT } \\
\text { PT/FT } \\
\text { PT/FT } \\
\text { FOU/PT/FT } \\
\text { FOU } \\
\text { PT } \\
\text { PT } \\
\text { PT/FT } \\
\text { PT } \\
\text { FOU } \\
\text { FOU }\end{array}$ & $\begin{array}{l}\text { Valine } \\
\beta \text {-D-OHbutyrate } \\
\text { Lactate } \\
\text { Alanine } \\
\text { Lysine } \\
\text { Arginine } \\
\text { Acetate } \\
\text { N-AC } \ddagger \\
\text { Glutamine } \\
\text { Succinate } \\
\text { Citrateft } \\
\text { Dimethylamine } \\
\text { Dimethylglycine } \\
\text { Creatinine } \\
\text { Creatine } \\
\text { Taurine } \\
\text { TMAO } \\
\text { Betaine } \\
\text { myo-inositol } \\
\alpha-G \text { lucose } \\
\alpha-G l u c o s e \\
\beta-G l u c o s e \\
\beta-G l u c o s e\end{array}$ & 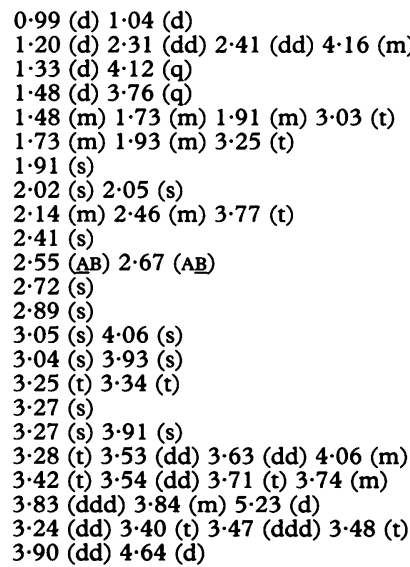 \\
\hline
\end{tabular}

FOU, fetus with obstructive uropathy; PT, preterm infant; FT, full term infant; TMAO, trimethylamine-N-oxide.

Data shown indicate the urinary metabolites that dominate, or are particularly prominent, in the one dimensional $500 \mathrm{MHz}{ }^{1} \mathrm{H}$ NMR spectra of fetal and neonatal urine.

${ }^{\star}$ Chemical shift of signal or centre of multiplet; tindicates form of peak and spin-spin coupling multiplicity; s, singlet; d, doublet; dd, doublet of doublets; $t$, triplet; q, quartet; m, multiplet;

$\mathrm{AB}$, non-equivalent two spin system, underlining indicates the relevant part of the spin system. $\neq \mathrm{AB}$, non-equivalent two spin system, underlining indicates the relevant part of the spin system. $\ddagger \mathrm{N}$-acetyl protons from various glycan moeities of $\mathrm{N}$-acetylated glycoproteins. H+Exact chemical
shift and linewidth of signals varies according to $\mathrm{pH}$ and the concentration of $\mathrm{Ca}^{2+}$ and $\mathrm{Mg}^{2+}$.

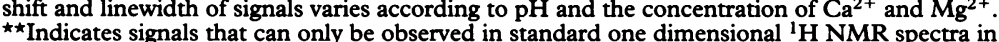
the absence of strong glucose signals.

urinary citrate, as observed in the normal neonates, is dependent on the ratio of citrate to metal ion $\left(\mathrm{Ca}^{2+}, \mathrm{Mg}^{2+}\right)$ concentration. Previous studies have shown that urinary $\mathrm{Ca}^{2+}$ does not change significantly with gestational age but that raised $\mathrm{Ca}^{2+}$ concentrations are associated with poor prognosis. ${ }^{2}$ Our observations that citrate was predominantly in the 'free' form in the fetal urines suggests that citrate concentrations are also increased, and in excess over those of the divalent metal ions, in cases of obstructive uropathy. These observations show that ${ }^{1} \mathrm{H}$ NMR can monitor dynamic interactions of metabolites in biological fluids, including chemical exchange phenomena and metal complexation reactions, and provide important, and hitherto unavailable, biochemical information on the developing kidney.

The ${ }^{1} \mathrm{H}$ NMR spectra of urine from preterm normal infants showed higher urinary concentration of taurine, myo-inositol, and TMAO with respect to creatinine than the full term urines (table 1). Those compounds, among their other functions, act as osmolytes and are present in high concentrations in the mammalian renal inner medulla. ${ }^{19-21}$ The intracellular accumulation of osmolytes protect medullary cells against the protein denaturing effect of their hyperosmolar external environment. The inner medullary content of myo-inositol and taurine is increased during antidiuresis and decreases during diuresis. ${ }^{20} 22$ Urinary concentrations of TMAO are increased in patients with renal allograft dysfunction, and it has been suggested that TMAO may be a marker of renal medullary damage. ${ }^{823}$ The results of this study lead to the hypothesis that urinary taurine, myoinositol, and TMAO are novel biochemical markers of medullary function in the human fetal kidney. Given that the excretion of these compounds was related to gestational age, we suggest that preterm infants would serve as useful controls for the evaluation of the later stages of normal fetal renal development. We emphasise, however, that it is the first urine passed - that is, urine produced during fetal life - that carries this important biochemical information and once feeding has been initiated the osmolyte composition changes accordingly.

Fetal obstructive uropathy is characterised by both functional and pathological changes caused by obstruction to the flow of urine and is associated with poor perinatal outcome. ${ }^{2-4}$ The assessment of renal function in utero is vital to determine prognosis and initiate appropriate treatment. The biochemical monitoring of fetal renal dysfunction is currently limited to the measurement of urine osmolality and electrolytes, including sodium and $\mathrm{Ca}^{2+}$, but interpretation of the results is controversial. ${ }^{2-4}$ The monitoring of obstructive uropathy in utero is, however, dependent on an understanding of normal fetal renal physiology in relation to gestational age. Due to the limited availability of normal fetal urine, the maturation of the fetal nephron and its function has largely been determined from changes in the volume and biochemistry of amniotic fluid. These are dependent on fetal micturition from 20 weeks of gestation. ${ }^{2}$ We have previously shown that the ${ }^{1} \mathrm{H}$ NMR spectra of amniotic fluid do not reflect closely the biochemical composition of fetal urine in cases of fetal obstructive uropathy. ${ }^{24}$ More recently, 400 $\mathrm{MHz}{ }^{1} \mathrm{H}$ NMR studies of fetal urine indicated that urinary alanine, threonine, and valine may be useful markers for the prediction of postnatal renal dysfunction in fetuses with bilateral uropathy. ${ }^{25}$ The observations were made retrospectively and based on good or bad renal outcome of fetuses at 1 year of life. Metabolic changes as a result of gestational age, and comparison with normal fetal renal function, were not primary considerations.

In the present study the ${ }^{1} \mathrm{H}$ NMR spectra of fetal urines from cases of obstructive uropathy were significantly different from those obtained for neonatal urines. Glycosuria, amino-aciduria (including raised alanine, and valine) and organic-aciduria were consistently observed in the obstructive uropathy group (fig 2). Whether these changes were related to the earlier gestational ages of the fetal group, and hence failure of solute reabsorption and secretion due to renal immaturity, rather than to renal damage is uncertain. Thus classification of birth outcome on the basis of the fetal urinary metabolic profiles would have been inappropriate in this study. However, the fetal urine obtained at 30 weeks' gestation (fig 2A) was significantly different in terms of glucose and amino acid composition from the preterm urines of similar gestational age. These observations confirm that samples of the first urine passed by normal preterm infants are useful controls for cases of obstructive uropathy detected in the third trimester.

${ }^{1} \mathrm{H}$ NMR spectroscopy provides a range of biochemical information that is not available 
with any other single analytical technique. Used in parallel with conventional clinical chemistry and histopathology ${ }^{1} \mathrm{H}$ NMR analysis of body fluids (such as urine, blood plasma, and cerebrospinal fluid) will increase our understanding of the biochemical basis of a wide range of disease processes. The close collaboration between clinicians and basic NMR scientists will lead, ultimately, to the widespread use of ${ }^{1} \mathrm{H}$ NMR in the clinical research laboratory. In this study we have shown that high field ${ }^{1} \mathrm{H}$ NMR based urinalysis studies are highly appropriate to the investigation of renal development in utero and in the assessment of fetal renal abnormalities and, as such, will become an important clinical diagnostic tool in the future.

We thank Dr J Kingdom, University College Obstetric Hospital, London, for valuable discussion, the ULIRS Hospital, London, for valuable discussion, the ULIRS
Biomedical $500 \mathrm{MHz}$ NMR service, and the St Peter's Research Trust and Siebe plc for financial support.
Remedical $500 \mathrm{MHz}$ NMR service, and the

1 Bard JBL, Woolf AS. Nephrogenesis and the development of renal disease. Nephrol Dial Transplant 1992; 7: 563-72. 2 Nicolini U, Fisk NM, Beacham J, Rodeck CH. Fetal urine biochemistry: an index of fetal maturation and dysfuncbiochemistry: an index of fetal maturation
tion. Br f Obstet Gynaecol 1992; 99: 46-50.

3 Lipitz S, Ryan G, Samuell C, Hausler MCH, Rodeck CH, Robson SC. Fetal urine analysis for the assessment of renal function in obstructive uropathy. $A m \mathcal{F}$ Obstet Gynecol 1993; 168: 174-9.

4 Crombleholme TM, Harrison MR, Golbus MS, Longaker MT, Langer JC, Callen PW. Fetal intervention in obstructive uropathy: prognostic indicators and efficacy of intervention. Am f Obstet Gynecol 1990; 162: 1239-44.

5 Videen JS, Ross BD. Proton nuclear magnetic resonance urinalysis: Coming of age. Kidney Int 1994; 46: S122-8.

6 Nicholson JK, Wilson ID. High resolution proton magnetic resonance spectroscopy of biological fluids. Prog NMR Spectroscopy 1989; 21: 449-501.

7 Foxall PJD, Bending MR, Gartland KPR, Nicholson JK. Acute renal failure following accidental cutaneous absorption of phenol: application of NMR urinalysis to monitor tion of phenol: application of NMR urinalysis to

8 Foxall PJD, Mellotte GJ, Bending MR, Lindon JC Nicholson JK. NMR spectroscopy as a novel approach to monitor renal transplant function. Kidney Int 1993; 43: 234-45.

9 Holmes E, Foxall PJD, Nicholson JK, Neild GH, Brown SM, Beddell CR, et al. Automatic data reduction and pattern recognition methods for analysis of ${ }^{1} \mathrm{H}$ nuclear magnetic resonance spectra of human urine from normal
and pathological states. Analyt Biochem 1994; 220: and pa

10 Foxall PJD, Price RG, Jones JK, Neild GH, Thompson FD, Nicholson JK. High resolution proton magnetic resonance spectroscopy of cyst fluids from patients with polycystic kidney disease. Biochem Biophys Acta 1992; 1138: 305-14.

11 Iles RA, Hind AJ, Chalmers RA. Use of proton nuclear magnetic resonance spectroscopy in detection and study of organic acidurias. Clin Chem 1985; 31: 1795-801.

12 Iles RA, Chalmers RA. Nuclear magnetic resonance spectroscopy in the study of inborn errors of metabolism. Clin Sci 1988; 74: 1-10.

13 Lehnert W, Hunkler D. Possibilities of selective screening for inborn errors of metabolism using high-resolution ${ }^{1} \mathrm{H}$ for inborn errors of metabolism using high-resolution ${ }^{1} \mathrm{H}-$

FT-NMR spectrometry. Eur f Pediatr 1986; 145: 260-6.
14 Nelson TR, Gillies RJ, Powell DA, Schrader MC, Manchester DK, Pretorius DH. High resolution proton NMR spectroscopy of human amniotic fluid. Prenat Diagn 1987; 7: 363-72.

15 Bell JD, Brown JC, Sadler PJ, Garvie D, Macleod AF, Lowy C. Maternal and cord blood plasma. Comparative analyses by ${ }^{1} \mathrm{H}$ NMR spectroscopy. NMR Biomed 1989; 2: 61-5.

16 Holmes E, Foxall PJD, Nicholson JK. Proton NMR analysis of plasma from renal failure patients: evaluation of sample preparation and spectral-editing methods. f Pharm Biomed Anal 1990; 8: 955-8.

17 Davies EC, Chalmers RA, Randall EW, Iles RA. Betaine metabolism in human neonates and developing rats. Clin Chim Acta 1988; 178: 241-50.

18 Davies SEC, Woolf DA, Chalmers RA, Rafter JEM, Iles RA. Proton NMR studies of betaine excretion in the
human neonate: consequences for choline and methylhuman neonate: consequences for choline and

19 Balaban RS, Burg MB. Osmotically active organic solutes in the renal inner medulla. Kidney Int 1987; 31: 562-4.

20 Bagnasco S, Balaban R, Fales HM, Yang YM, Burg $M$. Predominant osmotically active organic solutes in rat and rabbit renal medullas. $\mathcal{F}$ Biol Chem 1986; 261: 5872-7.

21 Sizeland PCB, Chambers ST, Lever M, Bason LM, Robson RA. Organic osmolytes in human and other mammalian kidneys. Kidney Int 1993; 43: 448-54.

22 Nakanishi T, Uyama O, Sugita $M$. Osmotically regulated taurine content in rat renal inner medulla. Am $\mathcal{F}$ Physiol 1991; 261: F957-63.

23 Le Moyec L, Pruna A, Eugene M, Bedrossian J, Idatte JM, Huneau JF, et al. Proton nuclear magnetic resonance spectroscopy of urine and plasma in renal transplantation spectroscopy of urine and plasma in
follow-up. Nephron 1993; 65: 433-9.

24 Foxall PJD, Bewley S, Robson SC, Rodeck CH, Neild GH, Nicholson JK. High resolution NMR spectroscopic measurements of fetal urine: A novel method to monitor renal abnormalities in utero. $\mathcal{f} \mathrm{Am}$ Soc Nephrol 1992; 3: 468.

25 Eugene M, Muller F, Dommergues M, Le Moyec L, Dumez Y. Evaluation of postnatal renal function in fetuses with bilateral obstructive nephropathies by proton nuclear magnetic resonance spectroscopy. Am f Obstet Gynecol 1994; 170: 595-602. 